\title{
Corrigendum
}

\section{Corrigendum to "Modeling Inhibitory Effect on the Growth of Uninfected T Cells Caused by Infected T Cells: Stability and Hopf Bifurcation"}

\author{
Yahui Ji, Wanbiao Ma ${ }^{(D)}$, and Keying Song \\ Department of Applied Mathematics, School of Mathematics and Physics, University of Science and Technology Beijing, \\ 100083 Beijing, China \\ Correspondence should be addressed to Wanbiao Ma; wanbiao_ma@ustb.edu.cn \\ Received 9 April 2019; Accepted 11 April 2019; Published 15 May 2019 \\ Copyright (C) 2019 Yahui Ji et al. This is an open access article distributed under the Creative Commons Attribution License, which \\ permits unrestricted use, distribution, and reproduction in any medium, provided the original work is properly cited.
}

In the article titled "Modeling Inhibitory Effect on the Growth of Uninfected T Cells Caused by Infected T Cells: Stability and Hopf Bifurcation" [1], there were several errors throughout the article which are as follows:

(i) In the Abstract and the Introduction sections, "infection-free" should be corrected to "uninfected."

(ii) In the Local and Global Stability of the Equilibria section, there were the following errors:

(1) "We can denote the basic reproduction number of the HIV virus for the model (3) as $R_{0}=(k \delta / p u) x_{0}$, $x_{0}=(T / 2 r)\left(r-d+\sqrt{(r-d)^{2}+4 s r / T}\right)$ (see, for example, [3])" should be corrected to "We can denote the basic reproduction number of the HIV virus for the model (3) as $R_{0}=(k \delta / p u) x_{0}$ (see, for example, [3])."

(2) The sentence "Proof. We consider linear system of the model (3) in $E_{0}$ near;" should be corrected to "Proof. We consider linear system of the model (3) at $E_{0} . "$

(3) The sentence "When $R_{0}<1, \quad p u-k \delta x_{0} \neq 0$. Therefore, $\lambda=0$ is not root of (9). If (8) has pure imaginary root $\lambda=i \omega(\omega>0)$ for some $\tau>0$, substituting it into (8) and separating the real and imaginary parts," should be corrected to "When $R_{0}<1, p u-k \delta x_{0} \neq 0$. Therefore, $\lambda=0$ is not root of (9). If (9) has pure imaginary root $\lambda=i \omega(\omega>0)$ for some $\tau>0$, substituting it into (9) and separating the real and imaginary parts."

(4) The sentence "Then, we get the corresponding $\tau_{k}^{(n)}>0$ such that (23) has pure imaginary $\lambda=i \omega_{k}$," should be corrected to "Then, we get the corresponding $\tau_{k}^{(n)}>0$ such that (19) has pure imaginary $\lambda=i \omega_{k}$."

(5) The sentence "From (19), we obtain $b_{2} \omega^{2}+b_{3}^{2}=\left(\omega^{3}-a_{2} \omega\right)^{2}+\left(a_{1} \omega^{2}-a_{3}\right)^{2 ”}$ should be corrected to "From (22), we obtain $b_{2}^{2} \omega^{2}+b_{3}^{2}=\left(\omega^{3}-a_{2} \omega\right)^{2}+\left(a_{1} \omega^{2}-a_{3}\right)^{2}$.

(6) Equation (L) should be corrected as follows:

$$
\begin{aligned}
{\left[\frac{d(\operatorname{Res}(\lambda))}{d \tau}\right]^{-1} \lambda=i \omega_{k} } & =\frac{3 v_{k}^{3}+2 c_{1} v_{k}^{2}+c_{2} v_{k}}{\omega_{k}^{2}\left[b_{2}^{2} \omega_{k}^{2}+b_{3}^{2}\right]} \\
& =\frac{v_{k} h^{\prime}\left(v_{k}\right)}{\omega_{k}^{2}\left[b_{2}^{2} \omega_{k}^{2}+b_{3}^{2}\right]} .
\end{aligned}
$$

(iii) In the Simulations and Conclusions section, the sentence "By direct calculations, we get that (19) has a positive root;" should be corrected to "By direct calculations, we get that (24) has a positive root."

(iv) The formats of references 2, 14, 25, 26, 27, and 29 were updated.

This has been corrected in place. 


\section{References}

[1] Y. Ji, W. Ma, and K. Song, "Modeling inhibitory effect on the growth of uninfected T cells caused by infected T cells: stability and hopf bifurcation," Computational and Mathematical Methods in Medicine, vol. 2018, Article ID 3176893, 10 pages, 2018. 


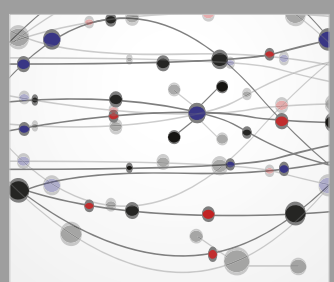

The Scientific World Journal
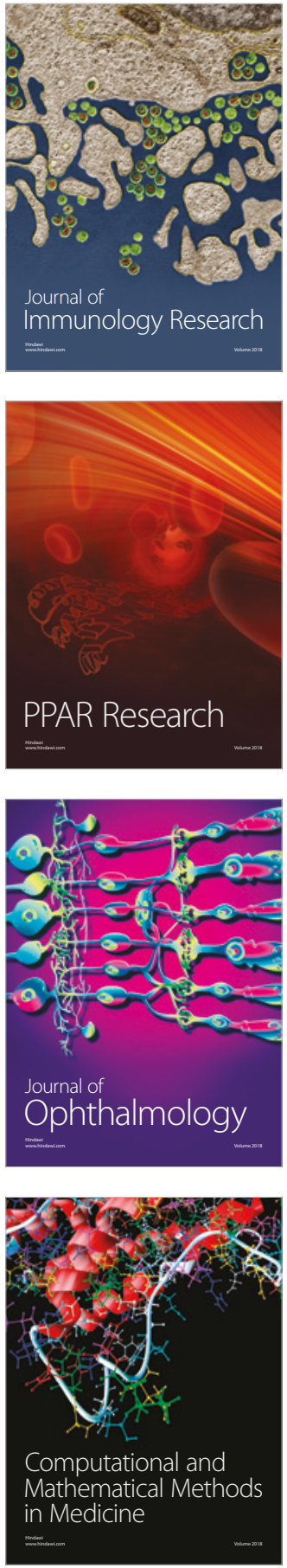

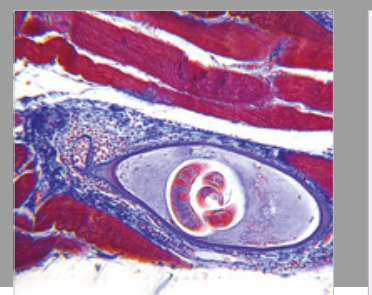

Gastroenterology Research and Practice

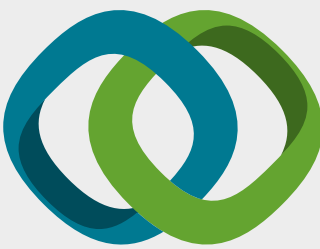

\section{Hindawi}

Submit your manuscripts at

www.hindawi.com
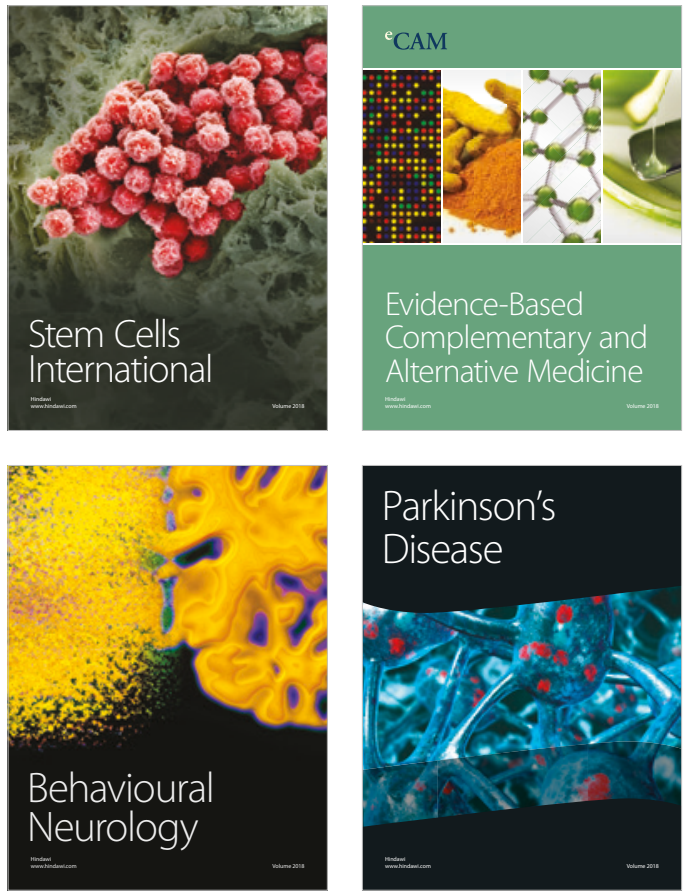

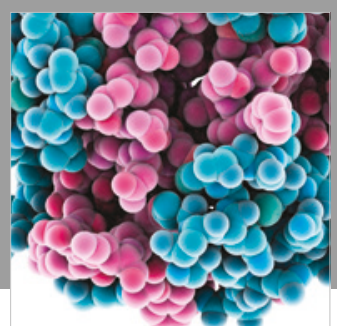

ournal of

Diabetes Research

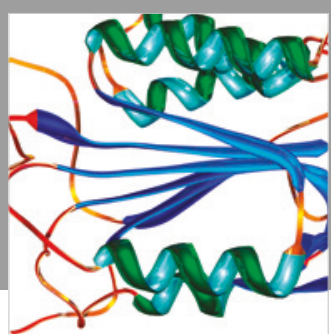

Disease Markers
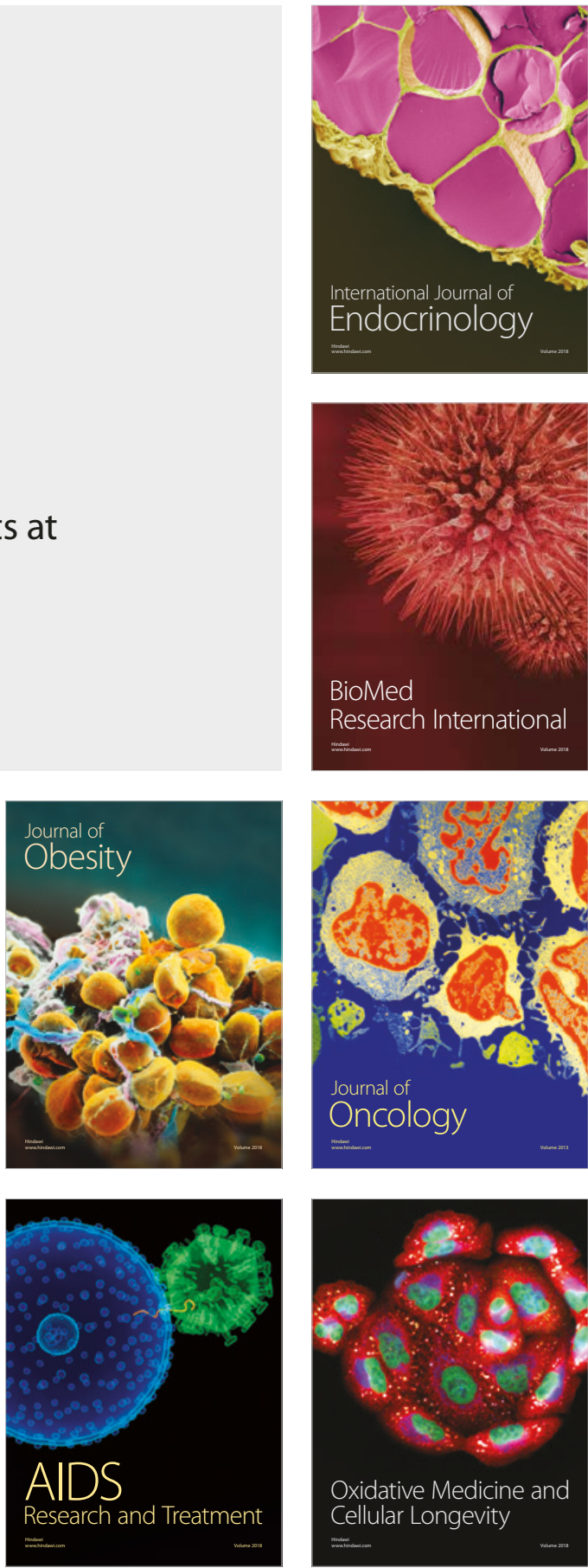